\title{
Synthesis and Spectrophotometric Properties of Sodium Metal Carboxylates
}

\author{
Iortyom Susan Doofan ${ }^{1}$, Kukwa Donald ${ }^{3}$, Iornumbe Esther Nguumbur ${ }^{2}$, Agbidye Isaac Gbaa ${ }^{3}$, \\ Ijuo Godwin ${ }^{2}$ \\ ${ }^{1}$ Department of Basic Sciences, Faculty of Basic and Health Sciences, Ernest Bai Koroma University of Science and Technology, Makeni, \\ Republic of Sierra Leone \\ ${ }^{2}$ Department of Chemistry, Federal University of Agriculture, Makurdi, Nigeria \\ ${ }^{3}$ Department of Chemistry, Benue State University, Makurdi, Nigeria
}

Email address:

iortyomsusan@gmail.com (I. S. Doofan)

\section{To cite this article:}

Iortyom Susan Doofan, Kukwa Donald, Iornumbe Esther Nguumbur, Agbidye Isaac Gbaa, Ijuo Godwin. Synthesis and Spectrophotometric Properties of Sodium Metal Carboxylates. Science Journal of Chemistry. Vol. 9, No. 5, 2021, pp. 113-120. doi: 10.11648/j.sjc.20210905.11

Received: July 28, 2021; Accepted: August 18, 2021; Published: September 6, 2021

\begin{abstract}
While metal carboxylates are by no means rare or inaccessible, complete characterization with notable exception of acetates is largely neglected hence a deficiency within the carboxylate literature giving rise to discrepancies within the characterization data available thus, spectroscopic properties of synthesized sodium metal carboxylates of acetic acid and myristic acid were investigated using Atomic Absorption Spectroscopy (AAS), flame photometry, FTIR spectrophotometer and UV- Visible spectrophotometry. AAS and flame photometric results showed elemental abundance of the sodium metals in the carboxylate complexes. FTIR spectra data revealed $v_{\mathrm{COO}}{ }^{-}$absorptions at $1636 \mathrm{~cm}^{-1}$ and $1558 \mathrm{~cm}^{-1}$ for sodium acetate and sodium myristate respectively. The free acid ligands showed absorptions at $1703 \mathrm{~cm}^{-1}$ for acetic acid which shifted to $1636 \mathrm{~cm}^{-}$ ${ }^{1}$ for sodium acetate. FTIR absorption of myristic acid revealed a strong absorption band at $1696 \mathrm{~cm}^{-1}$ which shifted to 1558 $\mathrm{cm}^{-1}$ in sodium myristate. The geometry of the complexes were determined by the magnitude of separation, $\Delta v$, which is equal to $v_{\text {aym }}-v_{\text {sym }}$. The values of $\Delta v$ for the synthesized sodium acetate complex was found to be $231 \mathrm{~cm}^{-1}$ and $138 \mathrm{~cm}^{-1}$ for sodium myristate indicating monodentate and bridging bidentate bonding respectively. Force constants of the carboxylates which correspond to their bond energies were found to be $1495.94 \mathrm{~K} \mathrm{NM}^{-1}$ and $1358 \mathrm{~K} \mathrm{NM}^{-1}$ for sodium acetate and sodium myristate, respectively. The UV- Visible analysis shows $K$ max values of $201 \mathrm{~nm}$ and $195 \mathrm{~nm}$ for sodium acetate and sodium myristate respectively, which corresponds to the absorption of the $\mathrm{COO}$ chromophore which is due to $\mathrm{n}-\pi^{*}$ transition.
\end{abstract}

Keywords: Carboxylates, Complexes, Bonds, Adsorption, Chromophore

\section{Introduction}

Many metal carboxylates have been known since ancient times. The first use of the material appeared to be in the formation of lead linoleates found in early paints used in mummification. The lubricating properties of these metal carboxylates were also noticed as early as 1400BC. From the dawn of the industrial revolution in the $18^{\text {th }}$ century, rapid progress in the field of metal carboxylates was made. Other uses then included water proofing materials and for adjusting the hardness of organic materials [1]. Metal carboxylates have emerged as an important family in the last few years. This family includes not only mono-and di- carboxylates of transition, rare-earth and main-group metals but also a variety of hybrid structures which possess novel adsorption and magnetic properties as well as spectral properties [2].

The chemistry of metal carboxylates continues to be an area of intense research in view of its diverse applications. Studies of metal carboxylates have been conducted to elucidate their physicochemical properties as well as applications in the chemical industry. In these studies, the structure of the carboxylate can apparently with great accuracy be predicted using their spectral, thermal and magnetic properties. Metal carboxylates exhibit fascinating structural features such as the structural diversity which is attributed to the versatile ligation behavior of the 
carboxylate group which can function like a bidentate ligand coordinating through two atoms or as monodentate ligand. In addition, the carboxylate ligand can stabilize polynuclear metal systems which exhibit interesting magnetic properties [3].

Chemically, metal carboxylates are regarded as derivatives of carboxylic acids which are organic Bronsted acids of the general formula $\mathrm{RCOOH}$ where $\mathrm{R}$ is a general organic moiety. The length and chemical nature (single or double bonds, linear or branched shapes, number and type of heteroatoms etc) of this organic residue determines the polarity and decomposition behavior of the acid and corresponding carboxylate. Short chain carboxylates are soluble in water whereas those of longer chains are not soluble in polar solvents due to increasing hydrophobic nature of the longer alkyl chain. These longer chain acids and their derivatives are soluble in less-polar solvents such as ethers, toluene etc [4].

The release of proton from carboxylic acid leads to the formation of the carboxylate anion which is stabilized by delocalization of the negative charge between the two oxygen atoms (resonance). Thus each of the carbon oxygen bonds in a carboxylate anion has a partial double-bond character which is also reflected in the carbon-oxygen bond lengths $(136 \mathrm{pm})$ that lies between the bond length of a carbonoxygen double bond (123pm) and single bond (143pm) [5]. Complex formation leads to several changes in the spectroscopic data of the complexed ligands relative to that of the free ligands. These could be due to changes in electronic structure or the state of symmetry of the metal carboxylate. These changes affect the vibrations of the complex causing a change in the vibrational spectrum of the metal carboxylate compared to that of the free ligand [6].

A widely used principle says that shifts in the wave numbers of carboxylate stretching mode upon bonding with a metal center can be used to determine the geometry of the metal carboxylates. Spectrophotometric techniques such as FTIR spectrophotometry, UV- Visible spectrophotometry and AAS have been found to be reliable techniques for this purpose.

In this study, sodium carboxylates of acetic acid and myristic acid were synthesized and characterized using AAS, FTIR, UV-Visible spectrophotometry and flame photometry.

\section{Materials and Methods}

Acetic acid, myristic acid, methanol, ethanol, acetone, DMSO and diethyl ether of high purity were purchased from Sigma Aldrich, Germany and used without further purification.

\subsection{Preparation of Sodium Carboxylates}

\subsubsection{Preparation of Sodium Acetate}

Acetic acid $(100 \mathrm{~mL})$ was poured into a $250 \mathrm{~mL}$ beaker containing sodium bicarbonate $(7 \mathrm{~g})$ little at a time until effervescence subsided. The solution was heated until about $20 \mathrm{~mL}$ of solution was remaining. The solution was poured into a watch glass and allowed to cool undisturbed to form long needle like crystals which were collected and dried in a desiccator. The dried crystals were collected and stored in an air tight container. The equation for the reaction is as shown in equation $[7,8]$.

$$
\mathrm{NaHCO}_{3}+\mathrm{CH}_{3} \mathrm{COOH} \rightarrow \mathrm{CH}_{3} \mathrm{COONa}+\mathrm{CO}_{2}+\mathrm{H}_{2} \mathrm{O}
$$

\subsubsection{Preparation of Sodium Myristate}

Sodium hydroxide pellets (10 g) was dissolved of $95 \%$ ethanol (100 $\mathrm{mL})$. Myristic acid (17 g) was added and the mixture heated to $68^{\circ} \mathrm{C}$ and refluxed for 20 minutes to form a goopy precipitate. Distilled water $(10 \mathrm{~mL})$ and saturated sodium chloride solution were added to precipitate the soap. The solution was filtered to collect a clumpy wet soap which was dried at room temperature for 2 hours to obtain a waxy white solid. This was collected, weighed and stored. The equation for the reaction is shown in equation [9].

$\mathrm{NaOH}+\mathrm{CH}_{3}\left(\mathrm{CH}_{2}\right)_{12} \mathrm{COOH} \rightarrow \mathrm{CH}_{3}\left(\mathrm{CH}_{2}\right)_{12} \mathrm{COONa}+\mathrm{H}_{2} \mathrm{O}$ (2)

\subsection{Determination of Percentage Yield}

The percentage yields of the synthesized metal carboxylates were calculated using the equation below;

$\%$ Yield $=$ Actual yield/Theoretical yield $\times 100$

\subsubsection{Determination of Solubility}

Supersaturated solutions of the synthesized metal carboxylates were made using distilled water, methanol, ethanol, acetone, DMSO and diethyl ether to determine the solubility of the metal carboxylates in the solvents.

\subsubsection{Determination of Melting Point}

Capillary tubes were filled with the solid samples of the prepared carboxylates to about $3 \mathrm{~mm}$ high. The tubes were then placed in a Barnstead Electro thermal melting point apparatus. The apparatus was set at a high level to make a rapid determination of melting point. The melting range was observed through the magnifying lens on the apparatus and recorded for each carboxylate.

\subsection{Spectral Characterization}

\subsubsection{FTIR Spectrophotometry}

The infrared spectra of the synthesized metal carboxylates were measured using a Shimadzhu FTIR8400s model and scanned between $4000 \mathrm{~cm}^{-1}$ and $700 \mathrm{~cm}^{-1}$ at 32 runs per minute. The charts were plotted on a computer inter-phased with the spectrophotometer and printed.

\subsubsection{Uv-Visible Spectrophotometry}

Uv- Visible analysis was carried out using a ShimadzhuUv2550 model spectrophotometer.

\subsubsection{Flame Photometry}

Sodium metal content in sodium acetate and sodium myristate was determined using flame photometry. 


\section{Results and Discussion}

\subsection{Yield and Percentage Yield}

The yield and percentage yield were calculated using equation (3) and tabulated for each complex as shown in Table 1. The synthesized complexes were obtained in good yield. The saponification route produced higher yields while the neutralization route produced metal carboxylates with lower yield especially for the acetates which were obtained as crystals.
Sodium acetate was obtained as white crystals of fine shape. This is in line with the findings of Groenewald and colleagues [9] and Tackett [10] in their respective investigations of metal acetates. Sodium myristates however was obtained as white solids as expected.

The metal carboxylates were synthesized using the direct reaction, classical acid-base and neutralization routes. Crystals and powdered products were obtained.

Table 1. Yield and Percentage yield for each complex.

\begin{tabular}{llll}
\hline Complex & Physical Appearance & Actual Yield & Theoretical Yield \\
\hline Sodium acetate & White needle like crystals & 30.00 & 143.00 \\
Sodium myristate & White granules & 17.73 & 31.25 \\
\hline
\end{tabular}

\subsection{Solubility Test of Metal Carboxylates in Some Solvents}

The results of solubility test carried out on the prepared sodium metal carboxylates are summarized in Table 2. This result showed that Sodium acetate was soluble in distilled water. This can be attributed to the polarity of the metal carboxylates and that of the solvents. Ionic or polar solutes dissolve in polar solvents. Non-polar solutes dissolve in non-polar solvents. Short chain carboxylates are soluble in water whereas those of longer chains are insoluble in water but slightly soluble in hot distilled water due to the increasing hydrophobic nature of the longer alkyl chain. Longer chain metal carboxylates are however soluble in nonpolar solvents [12].

\subsection{Melting Point of Metal Carboxylates}

Melting point range of the metal carboxylates which was determined using a Barnstead Electrothermal apparatus model 9100 are shown in Table 3 . The melting point range were observed to be $57^{\circ} \mathrm{C}-60^{\circ} \mathrm{C}$ for sodium acetate and $243^{\circ} \mathrm{C}-245^{\circ} \mathrm{C}$ for sodium myristates. These melting points are in close agreement with those obtained from literature indicating very minimal impurities in the prepared carboxylates. Shorter chain alkyl3 aliphatic compounds are of lower melting points as melting point increases with increasing carbon chain length of carboxylates. The increase in the melting point is however not linear as chain length increases. The complexes that have higher melting points are believed to have greater ionic character [13].
Table 2. Solubility of Metal carboxylates.

\begin{tabular}{lll}
\hline Solvent & Sodium acetate & Sodium myristate \\
\hline Methanol & IS & IS \\
Ethanol & IS & IS \\
Distilled $\mathrm{H}_{2} \mathrm{O}$ & $\mathrm{S}$ & $\mathrm{S}$ \\
Acetone & IS & IS \\
Diethyl ether & IS & IS \\
DMSO & IS & IS \\
\hline
\end{tabular}

KEY: $\mathrm{S}=$ soluble, $\mathrm{IS}=$ insoluble, $\mathrm{SS}=$ slightly soluble

Table 3. Melting point of metal carboxylates.

\begin{tabular}{ll}
\hline Complex & Melting Point $/{ }^{\circ} \mathbf{C}$ \\
\hline Sodium Acetate & $57-60$ \\
Sodium myristate & $107-110$ \\
\hline
\end{tabular}

\subsection{FTIR and Uv-Visible Spectra}

Frequencies of important functional groups in the free acid ligands and metal carboxylates are summarized in tables 4 and 5 , respectively.

The FTIR spectra of the free acid ligands display a strong band at $1703 \mathrm{~cm}^{-1}$ and $1654 \mathrm{~cm}^{-1}$ respectively for acetic acid and Myristic acid. These bands however shift to lower frequencies in the spectra of the corresponding metal carboxylates as can be shown in Figures $2(\mathrm{a}-\mathrm{d})$. Figures $3(\mathrm{a}-\mathrm{d})$ shows the comparison between the UvVisible absorptions due to the $\mathrm{COO}^{-}$chromophore in both the free acid spectra and their corresponding metal carboxylates.

\subsection{FTIR Spectra of Free Ligands}

Table 4. Frequencies for FTIR Spectra of Free Ligands.

\begin{tabular}{|c|c|c|c|c|}
\hline Ligands & $\tau\left(\mathrm{CH}_{3}\right) \mathrm{cm}^{-1}$ & $v(C=O) \mathrm{cm}^{-1}$ & $v\left(\mathrm{CH}_{3}\right) \mathrm{cm}^{-1}$ & $v(\mathrm{OH}) \mathrm{cm}^{-1}$ \\
\hline Acetic Acid & $887 \mathrm{~m}$ & $1730 \mathrm{~S}$ & $2899 w$ & $3384 \mathrm{~m}$ \\
\hline Myristic Acid & $723 w$ & $1654 w$ & $2918 \mathrm{~s}$ & $3399 b$ \\
\hline
\end{tabular}

$\mathrm{KEY} ; \mathrm{w}=$ weak band, $\mathrm{s}=$ strong band, $\mathrm{m}=$ medium band, $\tau=$ rocking vibration, $\mathrm{b}=$ broad band.

The IR spectra of acetic acid from table 4 displays a strong absorption band at $1703 \mathrm{~cm}^{-1}$ which corresponds to a carbonyl stretch $v(\mathrm{C}=\mathrm{O})$. A weak band at $2899 \mathrm{~cm}^{-1}$ is assigned to $v(\mathrm{C}-\mathrm{H})$ stretching frequencies and a strong broad band at $3384 \mathrm{~cm}^{-1}$ indicates the presence of the strongly bonded $v(\mathrm{O}-\mathrm{H})$ of carboxylic acids. A sharp absorption band at $1263 \mathrm{~cm}^{-1}$ is assigned to $v(\mathrm{C}-\mathrm{O})$ stretching vibration. The infrared spectrum of myristic acid (Figure 2) on the other hand shows a strong absorption band at $1696 \mathrm{~cm}^{-1}$ which is assigned $v(\mathrm{C}=\mathrm{O})$ stretch. A strong absorption band at 2914 
$\mathrm{cm}^{-1}$ is assigned to $v(\mathrm{C}-\mathrm{H})$ stretch. A very weak band at 2955 $\mathrm{cm}^{-1}$ is assigned to $\mathrm{O}-\mathrm{H}$ stretching frequency. Carboxylic acids form strongly bonded hydrogen dimers as the hydroxyl group has direct interaction with the carbonyl group in the condensed phase and this shifts the $(\mathrm{O}-\mathrm{H})$ stretching frequency to lower values of less than $3000 \mathrm{~cm}^{-1}$ depending on the strength of hydrogen bonding [14].

The presence of medium intensity methylene (twisting) band at $1446 \mathrm{~cm}^{-1}$ and another at $723 \mathrm{~cm}^{-1}$ (methylene rocking) vibration in the myristic acid spectrum indicates a long chain saturated aliphatic structure. These bands are however absent in the acetic acid spectrum indicating a short chain aliphatic structure [15]. The medium intensity band at $1237 \mathrm{~cm}^{-1}$ can also be assigned to $v(\mathrm{C}-\mathrm{O})$ stretching vibrations.

\subsection{FTIR Spectra of Metal Acetates}

FTIR spectra of metal carboxylates are shown in Figures 2 (a-d). The assignment of absorption bands to important functional groups is summarized in table 5. Upon complexation to the divalent metal centres, the absorption band of $v(\mathrm{C}=\mathrm{O})$ stretch in acetic acid at $1703 \mathrm{~cm}^{-1}$ which is on the high side due to extensive H-bonding in the carboxylic acid, shifted to $1636 \mathrm{~cm}^{-1}$ and $1408 \mathrm{~cm}^{-1}$ in sodium acetate spectrum. This occurs because, on complexation, $v(C=O)$ shifts to lower energy as its vibrational mode becomes coupled to that of the other oxygen giving rise to an asymmetric feature $\mathrm{V}_{\text {asy }}$. Similarly, the $(\mathrm{C}-\mathrm{OH})$ band shifts to higher energy on deprotonation yielding a symmetric $\mathrm{COO}^{-}$mode $\left(v_{\mathrm{s}}\right)$. This occurs as a result of the presence of a resonance stabilized carboxylate anion which contains two identical $\mathrm{C}=\mathrm{O}$ bonds of lower frequency due to the delocalization of the charge. Resonance or mesomeric effect also results in the decrease of the bond order of the $\mathrm{C}=\mathrm{O}$ bond leading to lower $\mathrm{v}(\mathrm{C}=\mathrm{O})$ stretch. The bonding in metal carboxylates involves electrons in the $\pi$ orbitals of the metal. Resonance interaction with electrons in the carbonyl group leads to a reduction in bond character of the carboxylate group. This reduces the force constant resulting in a lower wave number of absorbance compared to that of the free acids [16]. The lowering of the acetic acid carbonyl stretch occurs as bond orders of the carboxylate ion of both $(\mathrm{C}=\mathrm{O})$ and $(\mathrm{C}-\mathrm{OH})$ group tend towards each other as there is $\pi$-electron delocalization between the metal centre and $\mathrm{C}=\mathrm{O}$. This confirms that complex formation is through the carbonyl oxygen. Broad overlapping absorption bands at $3380 \mathrm{~cm}^{-1}$ and $3265 \mathrm{~cm}^{-1}$ in sodium acetate is assigned to $(\mathrm{O}-\mathrm{H})$ stretching vibrations which indicate that the complexes are hydrated. Although it is expected that upon formation of a new complex, the $(\mathrm{O}-\mathrm{H})$ stretch is supposed to disappear but the presence of water of crystallization accounts for the $(\mathrm{O}-\mathrm{H})$ vibration band observed [17].

Table 5. FTIR Spectra of Metal Carboxylates.

\begin{tabular}{|c|c|c|c|c|c|}
\hline Carboxylate & $v(\mathrm{COO})_{\text {asym }}$ & $v(\mathrm{COO})_{\mathrm{sym}}$ & $v(C-O)$ & $v(\mathbf{O H})$ & $v(C-H)$ \\
\hline Sodium Acetate & 1636 & 1408 & $1278 \mathrm{~m}$ & $3380 \mathrm{~m}$ & - \\
\hline Sodium myristate & $1695 \mathrm{~s}$ & $1468 \mathrm{~m}$ & $1189 \mathrm{~m}$ & - & $2914 s$ \\
\hline
\end{tabular}

Key: $\mathrm{s}=$ Strong, $\mathrm{m}=$ Medium, $\mathrm{w}=$ Weak

The absorption band at $2899 \mathrm{~cm}^{-1}$ assigned to $v(\mathrm{C}-\mathrm{H})$ in acetic acid spectrum occurs at higher wave numbers of 3265 $\mathrm{cm}^{-1}$ in the sodium acetate spectrum.

A medium intensity band observed at $1386 \mathrm{~cm}^{-1}$ in the free acetic acid spectrum assigned to $v(\mathrm{C}-\mathrm{O})$ stretch shifts to lower wave numbers of $1278 \mathrm{~cm}^{-1}$ in sodium acetate spectrum. This is also an indication that coordination is via oxygen atom with metal ion resulting in the formation of $\mathrm{C}$ $\mathrm{O}-\mathrm{M}$ bond. An ionic carboxylate is resonance stabilized through the delocalization of charge within the carboxylate group. This lowers the double bond character of the carbonyl group and consequently its force constant. The frequency of absorption of the carboxylate vibration is therefore expected at positions lower than seen in the ligands. This lowering in frequency can be attributed to steric hindrance due to the bulky nature of the ligand $[18,19]$.

\subsection{FTIR Spectra of Metal Myristates}

Just like in acetic acid, the $v\left(\mathrm{COO}^{-}\right)$stretching band of the myristic acid shifts to lower wave number due to coordination, to $1695 \mathrm{~cm}^{-1}$ and $1468 \mathrm{~cm}^{-1}$ in sodium myristate, corresponding to asymmetric and symmetric stretching vibrations of $v(\mathrm{COO})$. This shift is an indication that coordination occurs via the carbonyl oxygen atom of the carboxylate anion in the process of complex formation as well as the lowering of the COO bond character upon complex formation. The band assigned to $(\mathrm{O}-$ $\mathrm{H})$ stretch is absent in the spectra of sodium myristate. This indicates deprotonation and coordination and also that the complexes are anhydrous. Methylene rocking bands appear at $715 \mathrm{~cm}^{-1}$ in sodium. This is a consequence of inter chain vibrational coupling. The presence of COO- group reduces symmetry of the carboxylate chain compared to that of an alkane hence allowing more IR modes [20].

From the IR spectra of the metal carboxylates it is observed that, $\Delta v\left(v_{\text {asym }}-v_{\text {sym }}\right)$ is dictated mainly by the nature of the ion bound to the carboxylate. The same pattern was observed for the myristates being $130 \mathrm{~cm}^{-1}$ respectively for sodium. Apparently as the ionic character of the C-O bond increases, $\Delta v$ values increase. Therefore, apart from depicting the type of coordination of the metal carboxylate group in metal carboxylates, the magnitude of $\Delta v$ also indicates the electropositive character of the metal atom involved in the $\mathrm{O}-\mathrm{M}$ bond $[21,22]$.

\subsection{Determination of Bonding Modes of Sodium Carboxylates}

By evaluating the magnitude of separation $(\Delta v)$ between the asymmetric and the symmetric stretching vibration of the 
carbonyl functional group, the bonding modes of the synthesized carboxylates were deduced as shown in table 6 .

Table 6. Bonding Modes of the Synthesized Carboxylates.

\begin{tabular}{lll}
\hline Metal Carboxylate & $\boldsymbol{\Delta}\left(\mathrm{COO}^{-}\right)\left(\mathrm{cm}^{-1}\right)$ & Bonding mode \\
\hline Sodium Acetate & 231 & Monodentate \\
Sodium myristate & 227 & Monodentate \\
\hline
\end{tabular}

$\Delta v \mathrm{COO}^{-}=$vasymmetric COO absorption - vsymmetric COO absorption

By substituting the acid proton with a Sodium ion $\left(\mathrm{Na}^{+}\right)$, sodium carboxylates are formed, thus according to their chemical structure, the carboxyl group acts as a bidentate ligand either in a chelating or bridging mode. The difference in the magnitude of separation between the asymmetric and symmetric stretches of prepared the sodium carboxylates and their respective bonding modes are shown in table 6 . The $\Delta v(\mathrm{COO})$ values for the sodium carboxylates include, 231 $\mathrm{cm}^{-1}$ for Sodium acetate and $227 \mathrm{~cm}^{-1}$ for Sodium myristate, [23]. $\mathrm{Na}-\mathrm{O}$ bonds have a highly ionic character which explains the large value for the $\Delta v$ corresponding to ionic coordination $[24,25]$.

For monodentate coordination, a divergence of $v_{\text {asym }}(\mathrm{COO})$ and $v_{\text {sym }}(\mathrm{COO})$ compared with the free ion is expected due to the decrease in the equivalence of the $\mathrm{C}-\mathrm{O}$ bonds. [26]

According to Nelson and co-workers [21], for chelating bidentate coordination, $\Delta v=100 \mathrm{~cm}^{-1}$ or less, for bridging bidentate coordination, $\Delta v=100-150 \mathrm{~cm}^{-1}$, for ionic coordination, $\Delta v=200 \mathrm{~cm}^{-1}$ or more. This high value results from the fact that ionic coordination removes the equivalence of the $\mathrm{C}-\mathrm{O}$ bond resulting in a higher value for $\mathrm{v}_{\text {asym }}(\mathrm{COO})$ and a lower value for $v_{\text {sym }}(\mathrm{COO})$ hence $\Delta v$ will be much greater than or equal to $200 \mathrm{~cm}^{-1}$.

From the $\Delta v$ values of the synthesized sodium carboxylates, their coordination modes are monodentate for sodium acetate and sodium myristate. A relatively high value of $\Delta \mathrm{vCOO}^{-}$in the sodium carboxylate spectra could be taken as an indication of the slightly stronger metal- oxygen bonding in rare earth carboxylates, [19, 27, 28]. In the chelating mode, the metal ion is centered between the two oxygen atoms of the carboxylate group and is attracted to both oxygen atoms. In the bridging mode however, each carboxylate oxygen atom coordinates to one metal ion [29]. The structures of the synthesized sodium carboxylates as deduced from their FTIR spectra is as proposed in Figure 1. (i)<smiles></smiles>

Sodium acetate trihydrate<smiles>O=C(CCCCC[124I])O[18OH]</smiles>

(ii) Sodium myristate
Figure 1. Structures of synthesized sodium carboxylates showing their bonding modes.

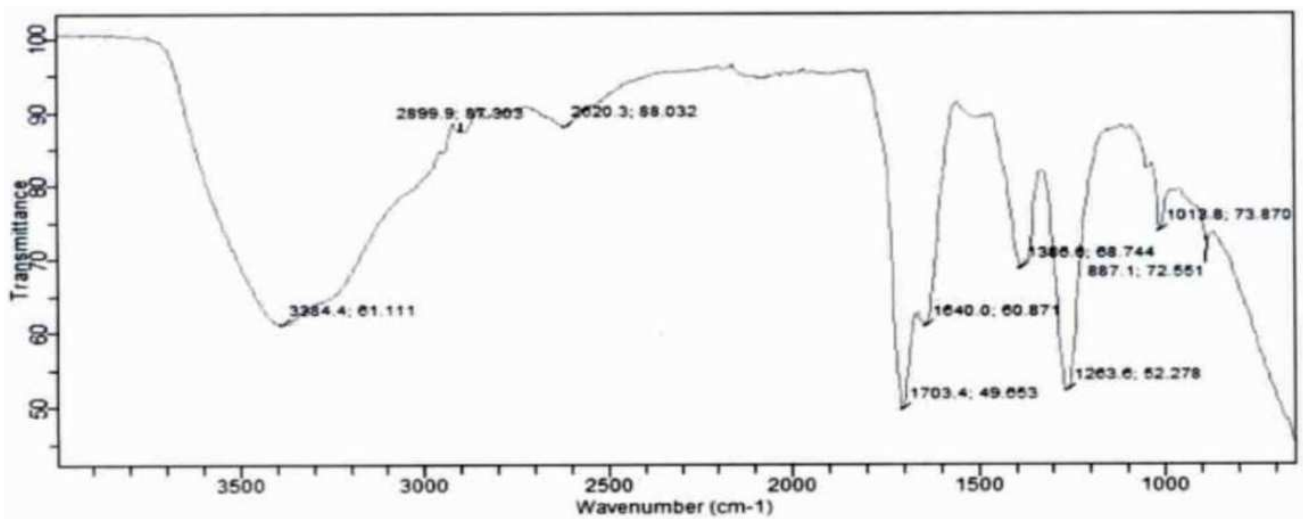

(a)

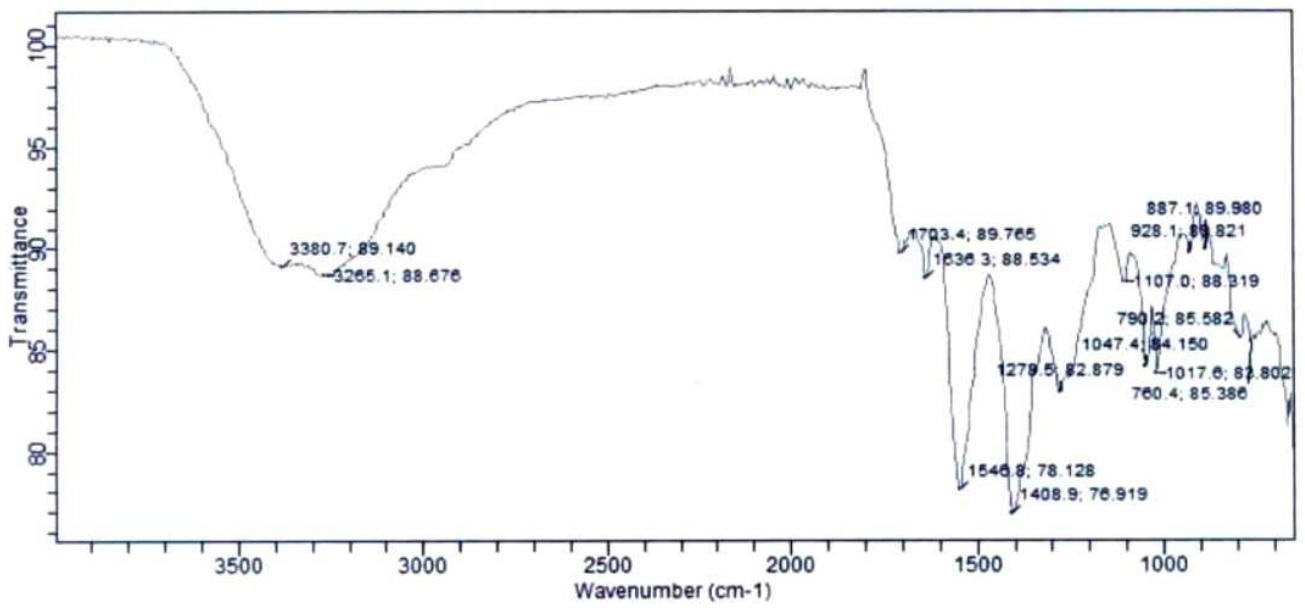

(b) 


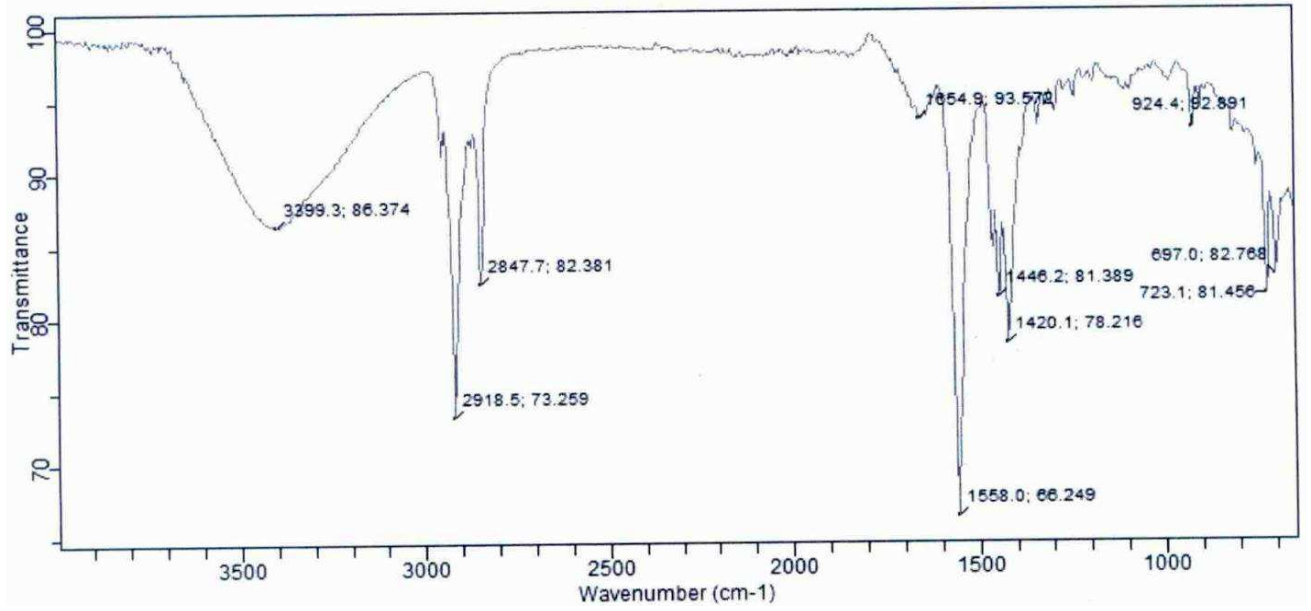

(c)

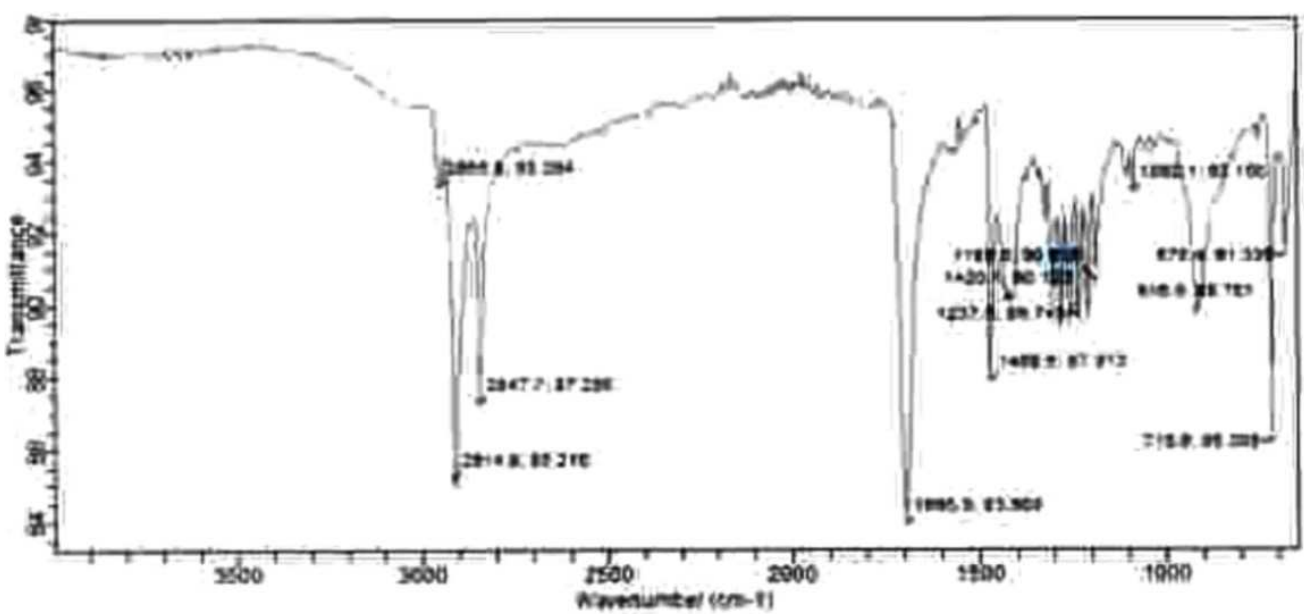

(d)

Figure 2. The FTIR spectra of (a) acetic acid and (b) sodium acetate (c) Myristic acid and (d) sodium myristate.

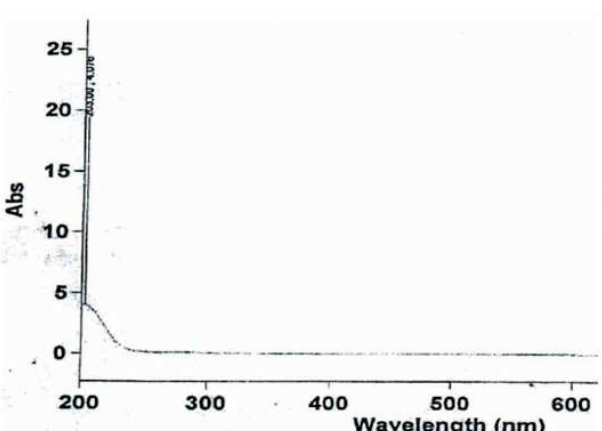

(a)

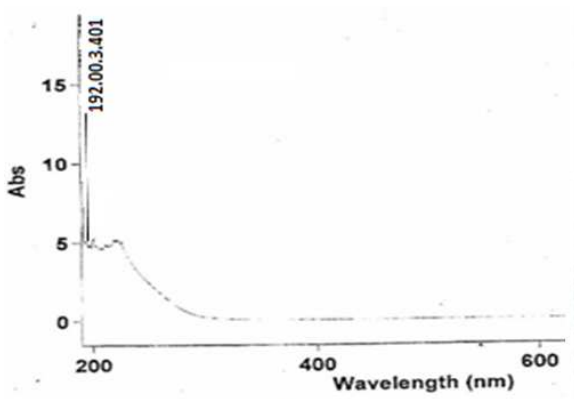

(c)

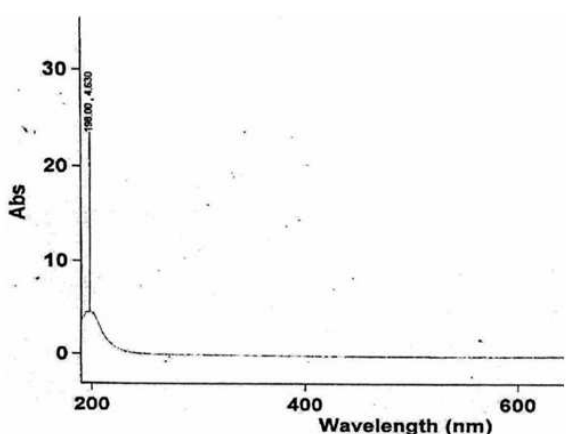

(b)

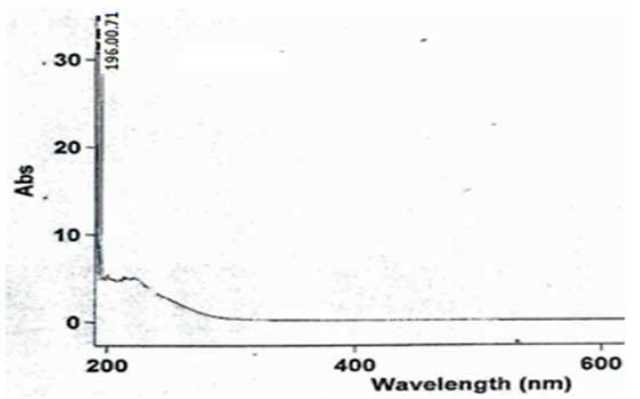

(d)

Figure 3. The Uv-Visible spectra of (a) acetic acid and (b) sodium acetate (c) Myristic acid and (d) sodium myristate. 
The bonding of these metal carboxylates can also be confirmed using difference in electronegativities of two bonded atoms. This provides a rough measure of the polarity to be expected in the bond and the bond type. For instance, non polar compounds are formed between atoms that have an electronegativity difference near zero, polar covalent compounds are formed between atoms with electronegativity difference of $0.4-1.8$ while for ionic compounds, the electronegativity difference greater than 1.8. The $\mathrm{Na}^{+} \mathrm{O}^{-}$bond is ionic and bi-polar with electronegative difference of 2.8. Electronegativity of elements is a tool for rationalizing bond energies, the types of reactions that substances undergo and the prediction of bond polarities. Highly electronegative atoms have a stronger tendency to acquire atoms hence the stronger the bonds it can form with a ligand [30].

\subsection{Atomic Absorption Spectral Data}

The atomic absorption spectrum which was used for determination of elemental content of the prepared metal carboxylates is given in table 7 .

Table 7. Elemental Content (AAS).

\begin{tabular}{llll}
\hline S/No & Complex & Metal & Conc.(g/kg) \\
\hline 1. & Sodium acetate & Sodium & 14.88 \\
4. & Sodium myristate & Sodium & 0.7912 \\
\hline
\end{tabular}

\subsection{Ultra violet - Visible Spectrophotometry and Electronic Spectra of Prepared Metal Carboxylates}

The results of the UV - visible spectroscopy revealed the absorptions due to the $\mathrm{COO}^{-}$chromophore. The maximum wavelength $(\lambda \max )$ and absorbance observed for the prepared metal carboxylates are given in the table 8 .

Table 8. Results of UV-visible Spectrophotometry.

\begin{tabular}{lll}
\hline Compound & $\lambda \boldsymbol{m a x}(\mathbf{n m})$ & Absorbance \\
\hline Sodium acetate & 201.00 & 4.435 \\
Sodium myristate & 195.00 & 10.00 \\
\hline
\end{tabular}

The electronic spectra of the ligands and their complexes were recorded in absolute ethanol for dibutyltin acetate and dibutyltin myristate and distilled water for sodium acetate and sodium myristate. The bands observed were assigned to $\mathrm{n}$ - (anti bonding) transitions according to their energies and intensities. It was observed that the electronic spectra of these complexes exhibit intense bands in the range of $183-230$ $\mathrm{nm}$ which is due to the forbidden $\mathrm{n}-\pi$ transitions of the (COO) chromophore [30].

\section{Conclusion}

This research was aimed at synthesis and characterization of sodium carboxylates of acetic acid and myristic acid. The findings showed that there was elemental abundance of the sodium metals in the carboxylate complex. Also, there was resonance between the metal center and $\mathrm{C}=\mathrm{O}$ as observed in the lowering of the $v_{\text {COO- }}$ absorption due to $\pi$ - electron delocalization. Monodentate and bidentate bonding was observed for sodium acetate complex and sodium myristate complex, respectively.

\section{Recommendations}

From this research we recommend the following,

1. The use of other techniques such as Raman spectroscopy and X- ray crystallography would verify and provide more information on the geometry of the carboxylates.

2. Principal component analysis can also be used as a tool in detecting the presence or lack of true band frequency shifts to explain spectra changes under temperature and concentration changes for carboxylates where $\mathrm{O}-\mathrm{H}$ stretching and $\mathrm{C}=\mathrm{O}$ stretching vibrations representing hydrogen bonding and dipole - dipole interactions are predominant.

\section{References}

[1] A. Pienaar (2005), Metal carboxylate Complexes Relevant to the Fischer- Tropsch Synthesis. (M.Sc. Thesis Chemistry and Polymer Science). University of Stellenbosch, pages 1-15.

[2] C. N. R Rao, S. Natarajan, R. Vaidyanathan (2004). Metal Carboxylates with Open Architecture. Angewandte Chemie Intenational. 43, 1466 - 1496.

[3] M. Tavakolli. (2011). Synthesis, Characterization and Antimicrobial Studies of Ruthenium (II) Carboxylates with 3 - Hydroxypyridine. International Journal of Chemistry. 3856 -860 .

[4] T. Schneller and D. Griesche (2013). Chemical Solution Deposition of Functional Oxides Thin Films. Springer verlagwien. Germany. 29-46.

[5] M. Peeran (2005). Effect of Coordination and Symmetry on The Spectra of Donor Molecules. www.chemvista.org.

[6] S. G. Yiase. (2003). Synthesis of Some Organotin and Silicon Compunds and Their Formulation as Antifungal Concentrates. (PhD THESIS. Chemistry dept ABU Zaria).

[7] Sodium Acetate- Wikipedia the free encyclopediahttp://en.m.wikipedia.com.

[8] C. S. Marcio and D. E. Nicoderm. (2002). Soap from Nutmeg: An integrated Introductory Organic Chemistry Laboratory Experiment. Journal of Chemical Education, 79 Pubs.acs.org/doi/abs/10.1021/ed079p94.

[9] G. S. Groenewold, W. de Jong, and M. J. Vanstipdonk. (2010): Ion Spectroscopy. Variable Denticity in Carboxylate Binding to Uranyl Coordination Complexes. Journal of the American Society for Mass Spectrometry. 21 (719-727).

[10] J. E. Tackett, (1989). FTIR Characterization of Metal Acetates in Aqueous Solutions. Journal of Applied Spectroscopy 43483-489.

[11] J. A. Telser, (1984), Synthetic and Spectroscopic Studies of Metal Carboxylate Dimers. A PhD Dissertation presented to the graduate school of the university of Florida. 
[12] M. Sherban-Kline, (2013). Infrared and Raman Spectroscopy: A key to Organic Structures. http/l. www.teacherinstituteyale.edu/curriculum/units/1999/s/99.05.0 7.x.hf.

[13] J. Coates, (2000). Interpretation of IR spectra: A Practical Approach. Encyclopedia of Analytical Chemistry. 10815 10837.

[14] F. Y Win, T. S Guan and B. M Yamin (2007). Synthesis and Characterization Of tributyltin (iv) Complexes of Pyridine Mono Carboxylic Acids. The Malaysian Journal of Analytical chemistry 35 (4): 285 - 294.

[15] K. S. Patel, C. S Patel, H. Dholariya, V. K. Patel and K. D. Patel, (2012). Synthesis of Cu (II), Ni (II), Co (II) and Mn (II) Complexes with Ciprofloxacin And Their Evaluation Of Antimicrobial, Antioxidant and Anti- tubercular activity. Open Journal of metal, $47-59$.

[16] B. Srinivasan, J. V. Savant, and P. Raghavaiah. (2006). Synthesis, Spectroscopy, Thermal / X-ray Structure Studies of Seven Coordinated Hydrate Ca (II) Parabenzoate showing Mono and Bidentate Carboxylate Ligation. Indian Journal of Chemistry. 45A, $2392-2399$

[17] M. Ibrahim. A. Nada. and D. E. Kamal, (2005). Density Functional theory and FTIR Spectroscopic study Of Carboxyl Group. Indian Journal of Pure and Applied Physics. 43, 911 917.

[18] Y. P. Win, S. Teoh, M. R. Vikneswaran, S. Ha, and P. Ibrahim, (2010). Synthesis and Characterization of Organotin (IV) Complexes Derived of (Diethylamino) benzoic acid: in- vitro Antibacterial Screening Activity. International Journal of the Physical Sciences 5, 1263- 1269.

[19] Infrared Spectrometry; The Nature Of Vibrational Spectrometry.

https://www2.Chemistry.msu,edu/faculty/reusch/virltxtjml/spe ctroscopy/infrared/irspec/html

[20] P. N. Nelson, and R. A. Taylor, (2014). Theories and Experimental Investigation of the Structural and Thermotropicmesomeric Phase Behavior of Metal Carboxylates. Journal of Applied Petrochemical Research. 4, $253-285$.
[21] X. J. Li, P. U. Guang, and F. Xi. (2012). Infrared Spectra of Dicarboxylates of Alkali Earth Metals. www.ncbi.nlm.nih.gov/m/pubmed/129383112

[22] D. A. Edwards and R. N Hayward (1968). Transition Metal Acetates. Canadian Journal of Chemistry 46: 3443 - 3450 .

[23] M. Kalinowska, R. Swislocka, L. Fuks, P. Koczon and W. Lewandowski. (2002). Spectroscopic studies on Metal Complexes with Benzoic acid and its Derivatives. Bioinorganic Chemistry and Applications.

[24] Ferenc W, Sadowski. P, Christovao B. and Sarzynski. Investigation of Some Properties of 4-Nitrocinnamates of Lanthanide (III). Journal of Chilean Chemical Society. 58: 265-270. Retrieved (5/06/2016).

[25] S. G. Yiase, M. S. Iorungwa, B. Ogoh, (2013). Synthesis, Spectral Characterization and Solubility Studies of Some Organotin Carboxylates. International Journal of Science and Research. $4666-670$.

[26] G. V. Sequel, B. L. Rivas and C. Nova, (2005). Polymeric Ligand - Metal Acetate Interactions. Spectroscopic Study and Semi Empirical Calculations. Journal of The Chilean Chemical Society 1- 6.

[27] W. Rehman, M. Kaleem, and A. Badshah, (2005). Comparative Study of Structure-Activity Relationship of Di and Tri Organotin (IV) Complexes of Monomethylglutarate. Journal of Brazilian Chemical Society. 16 http;//dx.doi.org/10.1590/s0103-505320005000500023.

[28] Y. P. Win, S. G. Teoh, and M. Y. Bohari, (2006). Synthesis and Characterization of Tributyltin (IV) Complexes Derived from Pyridine Monocarboxylic Acids. The Malaysian Journal of Analytical Sciences, 10, 285-294.

[29] M. Philip, (2003). Advanced Chemistry. Physical and Industrial. Cambridge. University Pres, 465 - 467.

[30] H. Husssain, V. U. Ahnad, I. R Green, K. Krohn, J. Hussain and A. Badshah., (2007). Antibacterial Organotin (IV) Compounds, Their Synthesis and Spectral Characterization. General online paper. ArKAT USA Inc. ISSN 1551-7012. 289 -299 . 